\title{
Antiplatelet Effects of Qishen Yiqi Dropping Pill in Platelets Aggregation in Hyperlipidemic Rabbits
}

\author{
Yi Wang, ${ }^{1,2}$ Jie Wang, ${ }^{1}$ Liping Guo, ${ }^{1}$ and Xiumei Gao ${ }^{1,2}$ \\ ${ }^{1}$ TCM Research Center, Tianjin University of Traditional Chinese Medicine, Tianjin 300193, China \\ ${ }^{2}$ Tianjin State Key Laboratory of Modern Chinese Medicine, Tianjin University of Traditional Chinese Medicine, \\ Tianjin 300193, China
}

Correspondence should be addressed to Xiumei Gao, gaoxiumei@tjutcm.edu.cn

Received 9 April 2012; Accepted 19 July 2012

Academic Editor: Alfredo Vannacci

Copyright (c) 2012 Yi Wang et al. This is an open access article distributed under the Creative Commons Attribution License, which permits unrestricted use, distribution, and reproduction in any medium, provided the original work is properly cited.

\begin{abstract}
We investigated the effects of Qishen Yiqi Dropping Pill (QSYQ) on platelets aggregation and its possible mechanisms. Hyperlipidemic model in rabbits was produced by a high fat/cholesterol diet for 6 weeks, the therapeutic effect of QSYQ with $2.0 \mathrm{~g} / \mathrm{kg}, 1.0 \mathrm{~g} / \mathrm{kg}$, and $0.5 \mathrm{~g} / \mathrm{kg}$ was observed. Fourteen days after drug treatment, platelet aggregation induced by adenosine diphosphate (ADP), arachidonic acid (AA), and collagen (COLL) was significantly reduced in rabbits of model group. Moreover, $\beta$-thromboglobulin $(\beta$-TG) level decreased obviously but no significant change in P-selectin and platelet factor 4 (PF4) level, while QSYQ significantly decreased the ratio of thromboxane $\mathrm{B} 2\left(\mathrm{TXB}_{2}\right)$ to 6-keto-prostaglandin $\mathrm{F}_{1 \alpha}$ (6-Keto-PGF $1 \alpha$ ) and increased cyclic adenosine monophosphate (cAMP) level in rabbits. In summary, QSYQ can improve platelets aggregation and inhibit the over-release of $\beta$-TG in hyperlipidemic rabbits; and the increased cAMP level may be involved in this process. These results suggest that the antiplatelet aggregation effect of QSYQ may be due to its ability to increase cAMP level for improving cAMP metabolism.
\end{abstract}

\section{Introduction}

It is universally acknowledged that thrombus formation affects the progression of various cardiovascular, cerebrovascular disorders and peripheral vascular diseases, including unstable angina, myocardial infarction, transient ischemic attack, and atherosclerosis [1]. Thrombin formation is a result of procoagulant and anticoagulant system imbalance, while platelets play a central role in the thrombus formation at the sites of vascular injury [2, 3]. According to the results of collaborative meta-analysis of randomized trials, antiplatelet therapy prevents serious vascular events such as nonfatal myocardial infarction, nonfatal stroke, and vascular death among a large number of patients at high risk for occlusive vascular event [4]. Therefore, agents with anti-platelet effects could become an important strategy for the treatment of circulatory diseases $[5,6]$. However, there are increasing reports in the recent decade that antithrombotic agents, such as aspirin, the thienopyridines (ticlopidine, clopidogrel), and the glycoprotein IIbIIIa antagonists (abciximab, eptifibatide) have side effects including gastrointestinal symptoms and hemorrhage [7-9]. Recently, antithrombotic agent discovered from medicinal plants with little side effects has attracted more attention $[7,10]$.

Qishen Yiqi Dropping Pill (QSYQ), made from Radix Astragalus membranaceus Bge, Radix Salvia miltiorrrhiza Bge, Pannax Notoginseng, and Dalbergia odorifera T. Chen, is a botanical drug used to treat and prevent chronic cardiac insufficiency, congestive heart failure, angina pectoris, and coronary heart disease in China [11-13]. Pharmacological studies showed that QSYQ can significantly reduce myocardial infarct size in left ventricle and accelerate angiogenesis in ischemic rats with left anterior descending artery ligation $[14,15]$. Recent studies also demonstrated that QSYQ decreased platelet aggregation induced by ADP and inhibited significantly the increase of $\left[\mathrm{Ca}^{2+}\right]$ produced from platelet activation in hyperlipidemic rabbits [16, 17]. However, the precise mechanism of antiplatelet effect of QSYQ has not been illustrated. The aim of the present study is to explore the possible mechanisms of QSYQ in the inhibition of platelet aggregation. 


\section{Materials and Methods}

2.1. Drugs and Reagents. QSYQ was provided by Tianjin Tasly Group Co. Ltd (Tianjin, China). Aspirin Enterie Ccoated Tablets was purchased from Bayer HealthCare AG (Leverkusen, Germany). Clopidogrel Sulfate Tablets were purchased from Sanofi-Aventis (Hangzhou, China). Adenosine diphosphate (ADP), AA, and collagen were purchased from Chrono-Log Co. (Havertown, PA, USA). ELISA kits for P-selectin, PF4, and $\beta$-TG were obtained from R\&D Systems Inc. (Minneapolis, MN, USA). RIA kits for thromboxan B2 (TXB2), 6-keto-prostaglandin, and cylic nucleotides radioimmunity were obtained from Beijing Sino-uk Institute of Biological Technology (Beijing, China). Cholesterol was obtained from Tianjin YingBo Biochemical Reagents Co. Ltd.

2.2. Animals. Male Big-Eared white rabbits (weight, approximately $2.0-2.5 \mathrm{~kg}$ ) were provided by Beijing Animal Breeding Center (certificate number SCXK (Beijing) 2003-0007). Animals were acclimated for at least 1 week in standard conditions and given free access to standard diet and tap water. All procedures were approved by the Animal Care and Use Committee of Tianjin University of Traditional Chinese Medicine and conform to the Guide for the Care and Use of Laboratory Animals published by the U.S. National Institutes of Health (NIH Publication number 85-23, revised 1996).

\subsection{Establishment of Hyperlipidemic Rabbit Model and Drug}

Treatment. 80 male rabbits were divided into two groups (control group and group A) according to the total cholesterol (TC) in plasma. Rabbits in control group (ten males) were fed a basic diet for 6 weeks. Rabbits in group A (70 males) were fed a high fat/cholesterol diet (1\% cholesterol, $10 \%$ arachis oil, $89 \%$ base animal feeds) for 6 weeks. The amount of daily diet for each animal was restricted to $50 \mathrm{~g}$ during the study period. Water was supplied ad lib. Six weeks later, their blood was collected from the ear edge vein.

60 hyperlipidemic rabbits in group A were selected by significantly higher TC value compared with control group and divided into six groups: model group, high dose QSYQ treatment group $(2.0 \mathrm{~g} / \mathrm{kg} /$ day $)$, middle dose QSYQ treatment group $(1.0 \mathrm{~g} / \mathrm{kg} /$ day $)$, low dose QSYQ treatment group $(0.5 \mathrm{~g} / \mathrm{kg} /$ day $)$, Aspirin treatment group $(3.5 \mathrm{mg} / \mathrm{kg} /$ day $)$, and Clopidogrel treatment group $(4.0 \mathrm{mg} / \mathrm{kg} /$ day $)$. Rabbits were given by oral administration and once daily consecutively for 14 days, respectively. Rabbits in these six groups except control group were still given high fat/cholesterol diet 6 times per week to maintain model.

2.4. Preparation of Washed Rabbit Platelets. Rabbits were local anesthetized with $2 \%$ lidocaine $(1 \mathrm{~mL})$. Blood was collected from the common carotid artery (CCA) and anticoagulated with citrate $(3.8 \% ; 1: 9$, v/v). Platelet-rich plasma (PRP) was obtained by centrifugation at $500 \mathrm{rpm}$ for $10 \mathrm{~min}$ and the remaining blood was further centrifuged at $3000 \mathrm{rpm}$ for $10 \mathrm{~min}$ to prepare platelet-poor plasma (PPP). After washed twice by $\mathrm{Ca}^{2+}$ free-Tyorde buffer, platelets were finally suspended in $\mathrm{Ca}^{2+}$-Tyorde buffer (containing $0.38 \%$
BSA). The platelet concentration was adjusted to $2 \times 10^{8}$ platelets/mL.

2.5. Determination of Platelet Aggregation. The level of platelet aggregation was measured using an aggregometer (570-VS, CHRONO-LOG, USA) according to the method reported by Born and Cross [18]. Briefly, $0.25 \mathrm{~mL}$ of PPP and PRP were placed in a cuvette, respectively, and stirred with rotor at $37^{\circ} \mathrm{C}$ for $5 \mathrm{~min}$; platelet aggregation was induced by the addition of ADP, AA, and COLL (final concentration, $13 \mu \mathrm{M}, 500 \mu \mathrm{M}$, and $10 \mathrm{mg} / \mathrm{L}$, resp.). Results were recorded as light transmission at maximal aggregation after the addition of an aggregating agent. Data are expressed as percent of maximal aggregation.

2.6. Determination of P-Selectin, PF4, and $\beta$-TG. Blood was collected from the CCA. After water bathed for $20 \mathrm{~min}$ at $37^{\circ} \mathrm{C}$, the serum was separated by centrifugation at $3500 \mathrm{rpm}$ for $10 \mathrm{~min}$. Levels of serum P-selectin, PF4, and $\beta$-TG were determined by the use of ELISA kits according to the manufacturers' instructions.

2.7. Determination of Plasma $\mathrm{TXB}_{2}, 6-$ Keto-PGF $\alpha$, and Cyclic AMP (cAMP) Level. Thromboxane $\mathrm{A}_{2}\left(\mathrm{TXA}_{2}\right)$ was evaluated by measuring its stable hydrolysis product, $\mathrm{TXB}_{2}$; prostacyclin $\left(\mathrm{PGI}_{2}\right)$ was measured as 6-keto-PGF $1 \alpha$ by radioimmunoassay. Blood was withdrawn from the CCA and collected into plastic tubes containing indomethacinEDTA $\cdot \mathrm{Na}_{2}$. Plasma was separated by centrifugation at $3500 \mathrm{rpm}$ for $15 \mathrm{~min}$. After measuring plasma samples by radioimmunoassay, $\mathrm{TXB}_{2}$ and 6-keto- $\mathrm{PGF}_{1 \alpha}$ levels were expressed as $\mathrm{pg} / \mathrm{mL}$ and cAMP levels were expressed as $\mathrm{pmol} / \mathrm{mL}$.

2.8. Statistical Analysis. All data were expressed as mean \pm SEM. Statistical analysis was performed using unpaired Student's $t$-test, ANOVA, and Kruskal Wallis test. $P<0.05$ was considered to be statistically significant. All statistical analyses were performed by SPSS11.5 (SPSS Inc.).

\section{Results}

3.1. Effects of QSYQ on Platelet Aggregation Induced by ADP, $A A$ and COLL. QSYQ exerted inhibitory effects on ADP, $\mathrm{AA}$ and COLL-induced platelet aggregation. As shown in Figure 1, compared with model group, high-dose group significantly reduced maximum gathered rate induced by ADP, AA, and COLL $(P<0.01)$, middle-dose group significantly reduced maximum gathered rate induced by ADP and COLL $(P<0.05)$, while low-dose group showed no significant result in maximum gathered rate. Aspirin and clopidogrel treatment group significantly reduced maximum gathered rate induced by ADP, AA, and COLL $(P<0.01)$.

3.2. Effects of QSYQ on P-Selectin, PF4, and $\beta$-TG Levels. Pselectin, PF4, and $\beta$-TG levels were determined by ELISA in this study. As shown in Table 1, the results showed that 


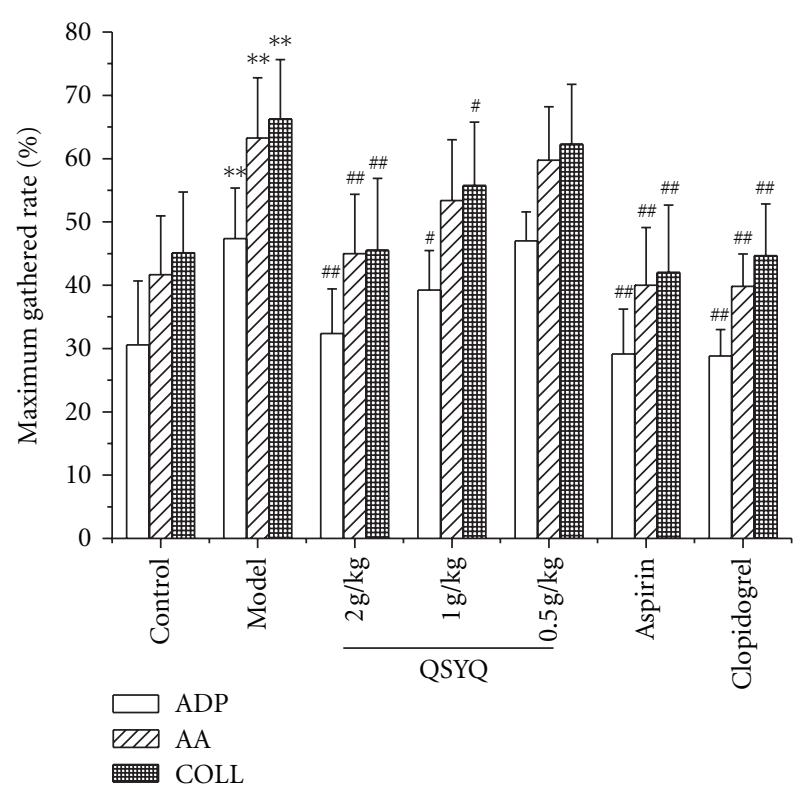

FIGURE 1: Effects of QSYQ on ADP, AA, and COLL-induced platelet aggregation. Blood was collected 1 hour after QSYQ intragastric administration and platelet aggregation was induced by ADP, AA, and COLL. Data is expressed as mean \pm SD (each group, $n \geqslant 6$ ). ${ }^{*} P<0.05,{ }^{*} P<0.01$ compared with control group; ${ }^{*} P<0.05$, ${ }^{\#} P<0.01$ compared with model group.

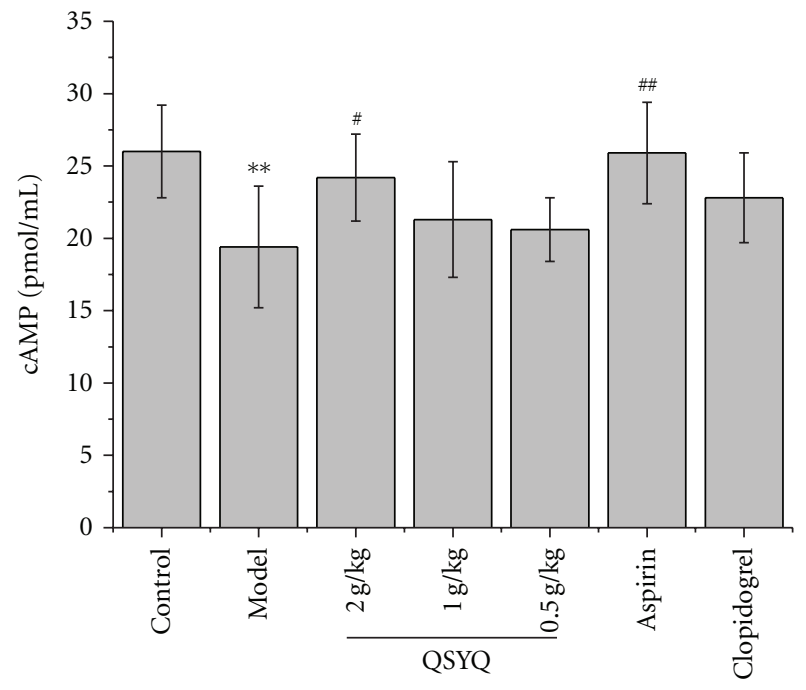

FIGURE 2: Effects of QSYQ on plasma cAMP levels in hyperlipidemic rabbits. Data are expressed as mean $\pm \mathrm{SD}$ (each group, $n \geqslant 6$ ). ${ }^{*} P<0.05,{ }^{* *} P<0.01$ compared with control group; ${ }^{*} P<0.05$, ${ }^{\# \#} P<0.01$ compared with model group.

compared with control group, model group increased significantly the levels of P-selectin, PF4, and $\beta$-TG. Compared with model group, high-dose group significantly decreased $\beta$-TG level but had no effect on P-selectin and PF4. Aspirin and clopidogrel treatment groups also showed apparent decrease in $\beta$-TG level.
TABLE 1: Effects of QSYQ on plasma P-selectin, PF4, and $\beta$-TG in hyperlipidemic rabbits.

\begin{tabular}{lccc}
\hline Groups & P-selectin $(\mathrm{ug} / \mathrm{L})$ & PF4 $(\mathrm{ug} / \mathrm{L})$ & $\beta$-TG $(\mathrm{ug} / \mathrm{L})$ \\
\hline Control & $2.80 \pm 1.04$ & $2.33 \pm 0.22$ & $40.78 \pm 8.91$ \\
Model & $3.73 \pm 1.56$ & $2.55 \pm 0.54$ & $59.74 \pm 8.91^{* *}$ \\
QSYQ 2.0 g/kg & $2.86 \pm 0.57$ & $2.16 \pm 0.50$ & $47.83 \pm 7.07^{\#}$ \\
QSYQ 1.0 g/kg & $3.23 \pm 1.43$ & $2.42 \pm 0.73$ & $52.44 \pm 15.27$ \\
QSYQ 0.5 g/kg & $3.18 \pm 1.24$ & $2.71 \pm 0.43$ & $52.19 \pm 11.33$ \\
Aspirin & $2.60 \pm 0.79$ & $2.25 \pm 0.24$ & $49.40 \pm 8.27^{\#}$ \\
Clopidogrel & $2.77 \pm 0.56$ & $2.17 \pm 0.50$ & $40.43 \pm 13.11^{\# \#}$ \\
\hline
\end{tabular}

Data is expressed as mean $\pm \mathrm{SD}$ (each group, $n \geqslant 7$ ).

${ }^{*} P<0.05,{ }^{* *} P<0.01$ compared with control group; ${ }^{\#} P<0.05,{ }^{\#} P<0.01$ compared with model group.

3.3. Effects of QSYQ on Plasma $T X B_{2}$ and 6-Keto-PGF $1 \alpha$. Compared with control, although 6-Keto-PGF $1 \alpha$ was significantly decreased, $\mathrm{TXB}_{2}$ and $\mathrm{TXB}_{2} / 6$-Keto-PGF $1 \alpha$ in plasma were markedly increased $(P<0.05)$ in model group. Levels of $\mathrm{TXB}_{2}, 6$-Keto-PGF $1 \alpha$, and $\mathrm{TXB}_{2} / 6-$ Keto- $\mathrm{PGF}_{1 \alpha}$ in high-dose group were significantly reduced from $101.91 \pm 8.52 \mathrm{pg} / \mathrm{mL}$, $309.33 \pm 45.24 \mathrm{pg} / \mathrm{mL}$, and $0.34 \pm 0.06 \mathrm{pg} / \mathrm{mL}$ to $100.08 \pm$ $10.33 \mathrm{pg} / \mathrm{mL}, 254.59 \pm 90.44 \mathrm{pg} / \mathrm{mL}$, and $0.46 \pm 0.23 \mathrm{pg} / \mathrm{mL}$, respectively. Aspirin and clopidogrel treatment groups could significantly decrease $\mathrm{TXB}_{2}$ and $\mathrm{TXB}_{2} / 6-\mathrm{Keto}^{-} \mathrm{PGF}_{1 \alpha}(P<$ $0.01 ; P<0.01)$ and increased 6-Keto-PGF $1 \alpha(P<0.05$, Table 2).

3.4. Effects of QSYQ on Plasma cAMP Level. Finally, we measured plasma cyclic AMP levels in hyperlipidemic rabbits. As shown in Figure 2, compared with control, cAMP level of platelet significantly decreased in model group $(P<0.01)$. cAMP levels with the treatment of QSYQ $(0.5,1,2 \mathrm{~g} / \mathrm{kg})$ were $24.2 \pm 3.0,21.3 \pm 4.0,20.6 \pm 2.2 \mathrm{pmol} / \mathrm{mL}$, respectively. The effect was significant when compared with $19.4 \pm$ $4.2 \mathrm{pmol} / \mathrm{mL}$ in model group. cAMP levels were significantly increased in Aspirin group $(P<0.05 ; P<0.01)$.

\section{Discussion}

There are many studies having been carried out worldwide to develop antiplatelet or antithrombotic agents with improved efficacy for preventing or treating arterial or venous thrombosis [7, 19]. Qishen Yiqi Dropping Pill has been most commonly used as a medicine for cardiovascular disease in China. However, there are rare researches on its antiplatelet activity and related mechanisms.

In present study, we evaluated the antiplatelet effects of QSYQ and the pharmacological mechanisms by which it inhibits platelet activation by evaluating its anti-platelet aggregative activity, antiplatelet release activity, and levels of arachidonic acid metabolic products and cAMP concentration. The findings from our study enable the better understanding of QSYQ, which could ultimately be helpful for the development of novel pharmaceutical strategies for the treatment of thrombosis diseases. 
TABLE 2: Effects of QSYQ on plasma $\mathrm{TXB}_{2}$ and 6-keto- $P G F_{1 \alpha}$ levels and the ratio of $\mathrm{TXB}_{2} / 6$-keto- $P G F_{1 \alpha}$ in hyperlipidemic rabbits.

\begin{tabular}{|c|c|c|c|}
\hline Groups & $\mathrm{TXB}_{2}(\mathrm{pg} / \mathrm{mL})$ & 6-Keto-PGF $1 \alpha(\mathrm{pg} / \mathrm{mL})$ & $\mathrm{TXB}_{2} / 6$-Keto-PGF $1 \alpha$ \\
\hline Control & $99.55 \pm 7.37$ & $336.84 \pm 52.17$ & $0.30 \pm 0.04$ \\
\hline Model & $109.98 \pm 6.10^{*}$ & $227.40 \pm 44.98^{* *}$ & $0.50 \pm 0.09^{* *}$ \\
\hline QSYQ $2.0 \mathrm{~g} / \mathrm{kg}$ & $101.91 \pm 8.52^{\#}$ & $309.33 \pm 45.24^{\# \#}$ & $0.34 \pm \pm 0.06^{\# \#}$ \\
\hline QSYQ $1.0 \mathrm{~g} / \mathrm{kg}$ & $100.08 \pm 10.33^{\#}$ & $254.59 \pm 90.44$ & $0.46 \pm 0.23$ \\
\hline QSYQ $0.5 \mathrm{~g} / \mathrm{kg}$ & $104.21 \pm 10.48$ & $255.27 \pm 89.87$ & $0.45 \pm 0.16$ \\
\hline Aspirin & $97.39 \pm 9.83^{\# \#}$ & $284.58 \pm 48.95^{\#}$ & $0.34 \pm 0.04^{\# \#}$ \\
\hline Clopidogrel & $104.15 \pm 5.42$ & $281.53 \pm 27.42^{\#}$ & $0.37 \pm 0.04^{\#}$ \\
\hline
\end{tabular}

Data are expressed as mean $\pm \mathrm{SD}$ (each group, $n \geqslant 6$ ).

${ }^{*} P<0.05,{ }^{*} P<0.01$ compared with control group; ${ }^{\#} P<0.05$, ${ }^{\# \#} P<0.01$ compared with model group.

Our results showed that QSYQ produced notable antiplatelet effects. We determined effects of QSYQ on platelet function by measuring the aggregation of washed platelets induced by various agonists (ADP, AA, and COLL). As shown in Figure 1, the experiments show that platelet aggregation can be significantly inhibited by QSYQ with $2.0 \mathrm{~g} / \mathrm{kg}$, and although QSYQ exerted only a slight inhibitory effect on platelet aggregation in middle dose group and lowdose group, the effect tendency was obvious. The present finding highlights that QSYQ may be investigated as a potential antiplatelet agent.

Platelets release is closely related to platelet aggregation, for instance, the second phase of platelet aggregation induced by ADP and 5-hydroxytryptamine (5-HT) is representative of the platelets release reaction, platelet aggregation induced by collagen is caused by the release of ADP in platelets [20]. Following vascular injury, the platelets are instantly activated by exposure to collagen. Activated platelets release containing factors to plasma and promote levels of P-selectin, PF4, and $\beta$-TG, which suggest the extent of platelet activation.

In our study, the results showed that QSYQ $2.0 \mathrm{~g} / \mathrm{kg}$ significantly decreased $\beta$-TG level but had no effect on Pselectin and PF4. The reason for this may be that although plasma lipoproteins increased in hyperlipemic rabbits, it could not reach the extent of promoting platelet activation, just making platelet keeping high sensitive to some physical stimuls.

Many factors involving in platelet functions, including adjusting arachidonic acid system, cAMP system, inositol phospholinid inositide signal system, and $\mathrm{Ca}^{2+}$. In our pervious study, we found plasma TC did not decrease significantly after QSYQ treatment, which demonstrated that antiplatelet effect of QSYQ is not achieved by reducing bloodfat [21]. Moreover, changes of $\left[\mathrm{Ca}^{2+}\right]_{i}$ in hyperlipemic rabbits after QSYQ treatment have been investigated, which showed that the increase of plasma $\left[\mathrm{Ca}^{2+}\right]_{i}$ may be one of mechanisms of thrombosis formation [17].

In the study, effect of QSYQ on arachidonic acid system, cAMP systems have been studied. TXA 2 is a powerful platelet aggregation agent, while the effect of $\mathrm{PGI}_{2}$ is just the opposite; the $\mathrm{TXA}_{2} / \mathrm{PGI}_{2}$ imbalance is one of the reasons which cause platelet aggregation and microcirculatory stasis [22]. Therefore, levels of $\mathrm{TXB}_{2}$ and 6-Keto-PGF $1 \alpha$ (metabolism products of $\mathrm{TXA}_{2}$ and $\mathrm{PGI}_{2}$ ) were measured. The results show that QSYQ can reduce $\mathrm{TXB}_{2}$ content in plasma of rabbits with hyperlipemia, increase 6-Keto-PGF $1 \alpha$ content, and adjust the balance between $\mathrm{TXA}_{2}$ and $\mathrm{PGI}_{2}$. Although the effect could not match with aspirin, it is stronger than clopidogrel in AA metabolism, therefore, it is suggested that adjusting the balance of $\mathrm{TXB}_{2}$ and 6-Keto-PGF $\mathrm{P}_{1 \alpha}$ may be one of mechanisms of QSYQ to inhibit platelet activation.

Cyclic nucleotide metabolic system including cAMP and cGMP, both of them are messenger materials. The cAMP can directly combined with $\mathrm{Ca}^{2+}$ and reduce the hydrolysis of phosphatidylinositol-4,5-bisphosphate $\left(\mathrm{PIP}_{2}\right)$, which inhibit the increase of $\mathrm{Ca}^{2+}$ induced by inositol triphosphate (IP3), and thereby reduce $\mathrm{Ca}^{2+}$ content in the platelet, inhibiting platelet aggregation. cAMP can also weaken the connection between thrombin and platelet, affecting protein kinase (PKC) activity to inhibit platelet gathering. The result shows that the QSYQ $2.0 \mathrm{~g} / \mathrm{kg}$ has the same affect with aspirin that can increase significantly the content of platelet cAMP in rabbits with hyperlipidemia, thereby playing the role of inhibiting platelet aggregation. It is suggested another probable mechanism of QSYQ to inhibit platelet activation.

In conclusion, the present results indicate that QSYQ shows a significant effect on platelet aggregation and platelet activation, and propose some possible mechanisms, which may provide useful clues to elucidating mechanism of action of QSYQ for the prevention and treatment of thrombotic disorders.

\section{Conflict of Interests}

The authors have declared that there is no conflict of interests.

\section{Acknowledgments}

This work was supported by the Grant from National Natural Science Foundation of China (no. 30830121, 30973935), and International S\&T Cooperation Project of China (no. 2009DFB30510). This project was also supported by the Program for Changjiang Scholars and Innovative Research Team in University, and the Foundation for the Author of National Excellent Doctoral Dissertation of China (no. 200779). 


\section{References}

[1] V. Fuster, P. M. Steels, and J. H. Chesebro, "Role of platelets and thrombosis in coronary atherosclerotic disease and sudden death," Journal of the American College of Cardiology, vol. 5, no. 6, pp. B175-B184, 1985.

[2] T. A. Meadows and D. L. Bhatt, "Clinical aspects of platelet inhibitors and thrombus formation," Circulation Research, vol. 100, no. 9, pp. 1261-1275, 2007.

[3] R. M. Bertina, "The role of procoagulants and anticoagulants in the development of venous thromboembolism," Thrombosis Research, vol. 123, no. 4, pp. S41-S45, 2009.

[4] C. Baigent, C. Sudlow, R. Collins, and R. Peto, "Collaborative meta-analysis of randomised trials of antiplatelet therapy for prevention of death, myocardial infarction, and stroke in high risk patients," British Medical Journal, vol. 324, no. 7329, pp. 71-86, 2002.

[5] S. F. De Meyer, K. Vanhoorelbeke, K. Broos, I. I. Salles, and H. Deckmyn, “Antiplatelet drugs," British Journal of Haematology, vol. 142, no. 4, pp. 515-528, 2008.

[6] J. M. Siller-Matula, J. Krumphuber, and B. Jilma, "Pharmacokinetic, pharmacodynamic and clinical profile of novel antiplatelet drugs targeting vascular diseases," British Journal of Pharmacology, vol. 159, no. 3, pp. 502-517, 2010.

[7] J. Lehár, A. S. Krueger, W. Avery et al., "Synergistic drug combinations tend to improve therapeutically relevant selectivity," Nature Biotechnology, vol. 27, no. 7, pp. 659-666, 2009.

[8] H. E. Speich, A. D. Earhart, S. N. Hill et al., "Variability of platelet aggregate dispersal with glycoprotein IIb-IIIa antagonists eptifibatide and abciximab," Journal of Thrombosis and Haemostasis, vol. 7, no. 6, pp. 983-991, 2009.

[9] C. P. Cannon, K. E. Rhee, R. M. Califf et al., "Current Use of Aspirin and Antithrombotic Agents in the United States Among Outpatients With Atherothrombotic Disease (from the REduction of Atherothrombosis for Continued Health [REACH] Registry)," American Journal of Cardiology, vol. 105, no. 4, pp. 445-452, 2010.

[10] K. H. Ryu, H. Y. Han, S. Y. Lee et al., "Ginkgo biloba extract enhances antiplatelet and antithrombotic effects of cilostazol without prolongation of bleeding time," Thrombosis Research, vol. 124, no. 3, pp. 328-334, 2009.

[11] W. Long, J. H. Luo, and G. H. Zhou, "Analysis of treatment of chronic cardiac insufficiency with Qishen yiqi drop pills," Journal of Jinggangshan Medical College, vol. 13, no. 1, p. 47, 2006.

[12] L. P. He and Y. F. Wu, "Clinical research on treating heart rate turbulence in chronic congestive heart failure with Qishen Yiqi dropping pills," Tianjin Journal of Traditional Chinese Medicine, vol. 26, no. 3, pp. 202-204, 2009.

[13] Y. X. Liao, Y. S. Wang, and H. L. Zhong, "Clinical observation of angina pectoris with coronary heart disease by using qishenyiqi dripping pills," China Journal of Modern Medicine, vol. 10, no. 2, 2008.

[14] B. L. Zhang, Y. Su, X. M. Gao, Z. L. Dong, Z. P. Xu, and X. H. Wang, "Clinical and experimental research of compound huangqi danshen granule on treating angina pectoris," Gianjin Journal of Traditional Chinese Medicine, vol. 19, no. 4, pp. 1215, 2002.

[15] L. Zhang, Y. Wang, L. Yu et al., "QI-SHEN-YI-QI accelerates angiogenesis after myocardial infarction in rats," International Journal of Cardiology, vol. 143, no. 1, pp. 105-109, 2010.

[16] J. Wang, L. P. Guo, Y. Wang, Y. Z. Song, and Wang y, "Effect of Qishen Yiqi dropping pill on platelet adhesion and aggregation in rabbits," Jilin Journal of Traditional Chinese Medicine, vol. 29, no. 3, 2009.

[17] J. Yuan, M. Yang, H. Yao et al., "Plasma antibodies to heat shock protein 60 and heat shock protein 70 are associated with increased risk of electrocardiograph abnormalities in automobile workers exposed to noise," Cell Stress and Chaperones, vol. 10, no. 2, pp. 126-135, 2005.

[18] G. V. Born and M. J. Cross, "The aggregation of blood platelets," The Journal of physiology, vol. 168, pp. 178-195, 1963.

[19] X. H. Zhang, Z. L. Lu, and L. Liu, "Coronary heart disease in China," Heart, vol. 94, no. 9, pp. 1126-1131, 2008.

[20] J. B. Smith and A. L. Willis, "Formation and release of prostaglandins by platelets in response to thrombin," British Journal of Pharmacology, vol. 40, no. 3, pp. P545-P546, 1970.

[21] L. Zhang, K. Yan, Y. Zhang et al., "High-throughput synergy screening identifies microbial metabolites as combination agents for the treatment of fungal infections," Proceedings of the National Academy of Sciences of the United States of America, vol. 104, no. 11, pp. 4606-4611, 2007.

[22] T. Mitsuhashi, T. Ikata, K. Morimoto, T. Tonai, and S. Katoh, "Increased production of eicosanoids, TXA2, PGI2 and LTC4 in experimental spinal cord injuries," Paraplegia, vol. 32, no. 8, pp. 524-530, 1994. 


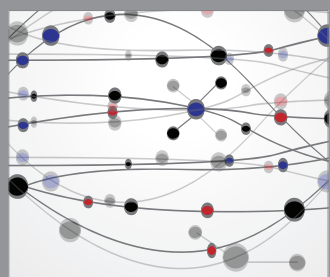

The Scientific World Journal
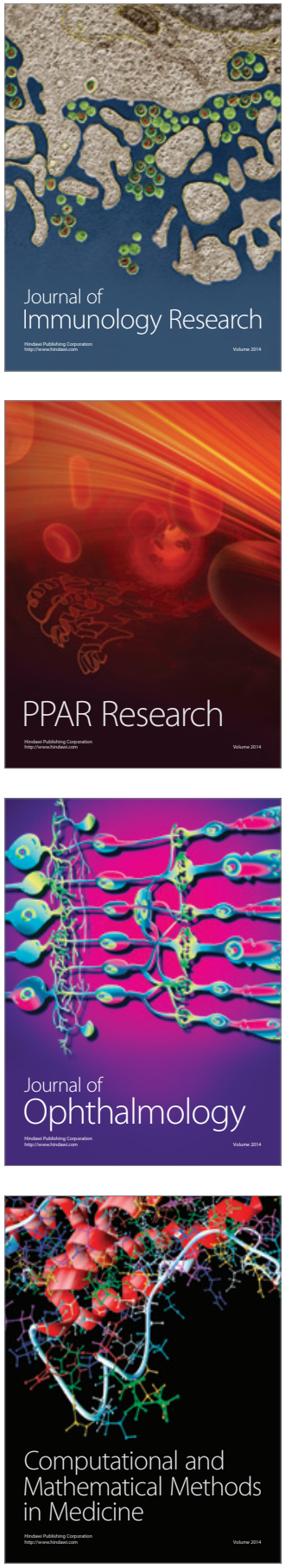

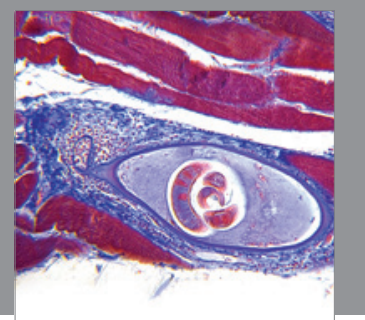

Gastroenterology

Research and Practice
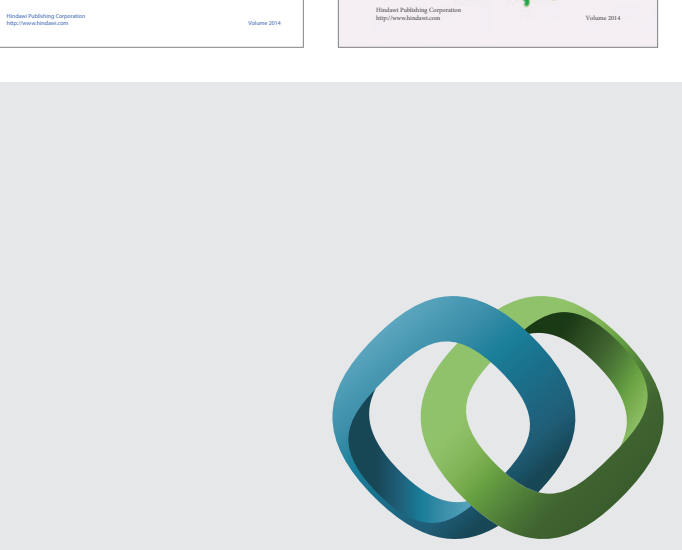

\section{Hindawi}

Submit your manuscripts at

http://www.hindawi.com
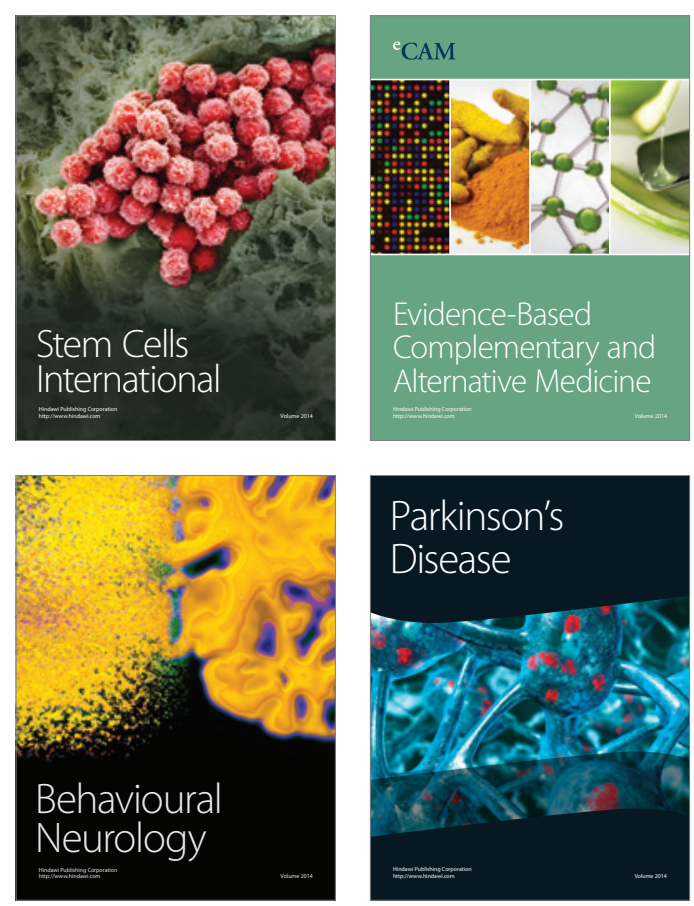

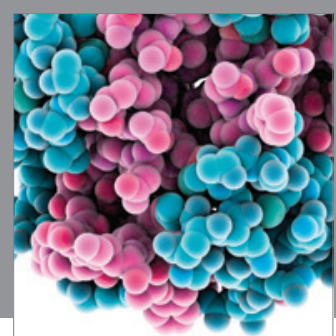

Journal of
Diabetes Research

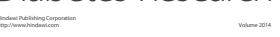

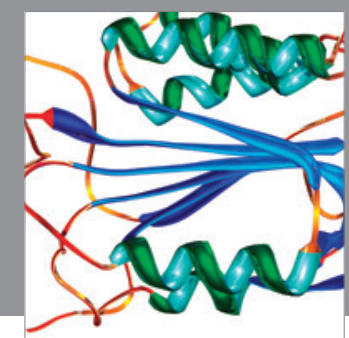

Disease Markers
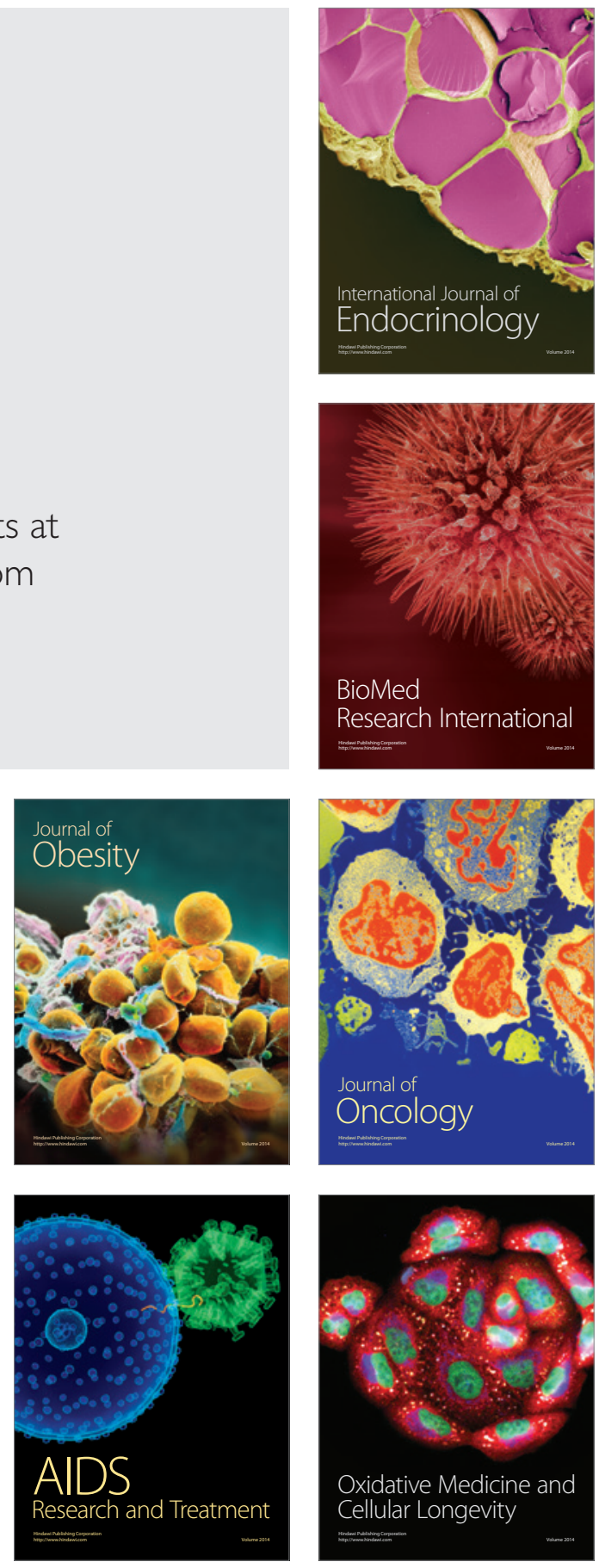